\title{
O Comércio Intraindustrial entre Brasil e os Países da OCDE: Decomposição e Análise de seus Determinantes
}

\section{Intra-industry Trade between Brazil and OECD Countries: Decomposition and Determinants Analysis}

\author{
Alex Sander Souza do Carmo* \\ Maurício Vaz Lobo Bittencourt**
}

Resumo: O objetivo principal do presente trabalho é analisar o comércio intraindustrial (CII) entre o Brasil e os países da OCDE, no período compreendido de 2000 a 2009. Especificamente, pretende-se decompor o comércio intraindustrial (CII) bilateral e analisar os seus determinantes. Os principais resultados encontrados foram: os Estados Unidos e o México são os países da OCDE que possuem o maior volume e índice de comércio intraindustrial (CII) com o Brasil, respectivamente. Ademais, verificou-se que, em todas as relações bilaterais, o comércio intraindustrial vertical (CIIV) é superior ao comércio intraindustrial horizontal (CIIH), em que os produtos exportados pelo Brasil possuem qualidade inferior à dos produtos importados. Após a decomposição do comércio intraindustrial (CII), partiu-se para a análise empírica dos seus determinantes. Conforme o resultado obtido a partir do método de efeitos aleatórios, verificou-se que, a 10\% de significância, a diferença na dotação de fatores (em termos da razão capital/trabalho) tem um impacto positivo sobre o comércio intraindustrial vertical bilateral, corroborando a hipótese central do modelo de Falvey e Kierzkowski (1987).

Palavras-chave: Comércio intraindustrial. Diferenciação de produtos. Painel de dados.

Abstract: The main goal of this paper is to analyze the intra-industry trade between Brazil and OECD countries for the period 2000 - 2009. Specifically, it is intended to decompose the intra-industry bilateral trade (CII) and find its determinants. The main results showed that the USA and Mexico have the largest volume and index

\footnotetext{
* Doutorando em Desenvolvimento Econômico pela Universidade Federal do Paraná (PPGDE/ UFPR). Professor assistente da Universidade Estadual de Ponta Grossa (UEPG). E-mail: acarmo@ uepg.br

* Doutor em Economia pela Ohio State University. Professor do Programa de Pós-Graduação em Desenvolvimento Econômico da Universidade Federal do Paraná (PPGDE/UFPR). Professor visitante na Ohio State University (EUA). Bolsista do CNPq. E-mail: mbittencourt@ufpr.br
} 
of CII with Brazil, respectively. Additionally, it was found that the vertical intra-industry trade (CIIV) is larger than the horizontal intra-industry trade (CIIH), where the Brazilian exports are mainly based on lower quality goods than imports. The econometric approach to investigate the main determinants of the CIIV was based on the theoretical model from Falvey and Kierzkowski (1987), through panel data econometrics. According to the results from the Random Effects estimations, with a $10 \%$ statistical significance, the coefficient for endowments differences (in terms of capital/labor ratio) has a positive effect on vertical intra-industry bilateral trade, which corroborates the main hypothesis of the Falvey and Kierzkowski (1987) model.

Keywords: Intra-industry trade. Products differentiation. Panel data.

JEL Classification: F10; F14; F15.

\section{Introdução}

Conforme a literatura (GREENAWAY; HINE; MILNER, 1994), os fluxos comerciais são divididos em dois distintos padrões: comércio interindustrial e comércio intraindustrial. Entende-se por comércio interindustrial uma situação em que dois parceiros comerciais trocam produtos de diferentes categorias industriais. Já o comércio intraindustrial ocorre quando os parceiros comerciais trocam simultaneamente produtos de uma mesma categoria industrial, ou seja, esse tipo de comércio se constitui em uma medida de similaridade da pauta de exportação entre dois parceiros comerciais.

Caso essa similaridade ocorra em produtos horizontalmente diferenciados (diferenciados em variedade), o comércio intraindustrial será entendido como horizontal (CIIH). Já na situação em que a similaridade ocorre em produtos verticalmente diferenciados (diferenciados por qualidade), o referido padrão de comércio será entendido como vertical (CIIV). Em tese, o comércio intraindustrial horizontal (CIIH) deve ocorrer principalmente entre parceiros comerciais com semelhantes níveis de renda, ao passo que o comércio intraindustrial vertical (CIIV) deve ocorrer entre países com distintos níveis de renda (EKANAYAKE et al., 2007).

Zhang, Van Witteloostuijn e Zhou (2005) aponta que na literatura internacional os estudos sobre o tema comércio intraindustrial são divididos em três fases. Na primeira, que corresponde aos anos 60 e 70, os estudos se concentraram sobretudo na identificação desse padrão de comércio e no desenvolvimento dos métodos de mensuração. Na segunda fase, que corresponde ao período dos anos 80 , a agenda de pesquisa se concentrou principalmente no desenvolvimento dos modelos teóricos. Na terceira fase, dos anos 90 em diante, as pesquisas voltaram- 
-se para a decomposição do comércio intraindustrial e para a análise dos seus determinantes. Particularmente, o presente artigo se enquadra na terceira fase.

A importância de decompor o comércio intraindustrial está relacionada ao fato de que o comércio intraindustrial horizontal (CIIH) e o vertical (CIIV) possuem distintos arcabouços teóricos. O comércio intraindustrial horizontal (CIIH) é explicado a partir da nova teoria de comércio internacional (KRUGMAN, 1981, 1980, 1979; LANCASTER, 1980; HELPMAN; KRUGMAN, 1985), que explica esse padrão de comércio a partir de modelos que admitem competição monopolística, diferenciação horizontal de produtos e retornos crescentes de escala. Já o comércio intraindustrial vertical (CIIV) é explicado a partir das tradicionais teorias de comércio internacional, que explicam esse padrão de comércio a partir das vantagens comparativas dos países (FALVEY, 1981; FALVEY; KIERZKOWSKI, 1987; FLAM; HELPMAN, 1987).

Sob o ponto de vista empírico, Gullstrand (2000) aponta que a formulação de um modelo econométrico que utilize como variável dependente a magnitude total de comércio intraindustrial, provavelmente, enfrentará problemas de variáveis omitidas. Isso porque, como salientado anteriormente, o comércio intraindustrial horizontal e o vertical estão relacionados a diferentes arcabouços teóricos e, sendo assim, devem possuir distintos determinantes. Na literatura internacional, são vários os trabalhos que objetivam analisar os determinantes do comércio intraindustrial horizontal e do vertical, entre os quais podemos destacar: Greenaway, Hine e Milner (1994), Durkin e Krygier (2000), Blanes e Martin (2000), Martin e Orts (2002), Crespo e Fontoura (2004), Zhang, Van Witteloostuijn e Zhou (2005), Baleix e Egidio (2005), Ekanayake et al. (2007) e Jansen e Lüthje (2009).

Especificamente para o Brasil, os estudos sobre o comércio intraindustrial emergiram na literatura no final dos anos 80. Lerda (1988 ${ }^{1}$ apud VASCONCELOS, 2003) calcula o comércio intraindustrial entre o Brasil e o resto do mundo e entre o Brasil e a Argentina no período entre 1981 a 1985. O estudo compreendeu todos os setores manufaturados. Nesse caso, verificou-se que a participação do comércio intraindustrial, em relação ao comércio total de produtos manufaturados, foi de $46 \%$ (em 1985), entre o Brasil e o resto do mundo, e de $35 \%$ (em 1985) entre o Brasil e a Argentina.

Hidalgo (1993) mensura o comércio intraindustrial entre o Brasil e o resto do mundo para o período compreendido entre 1978 e 1987 . Os resultados evidenciam que a participação do comércio intraindustrial em relação ao comércio total variou entre $30 \%$ a $40 \%$ durante o período analisado. Ademais, no mesmo trabalho, o autor avaliou os determinantes do comércio intraindustrial. Nesse caso, as

LERDA, S. S. Comércio internacional intra-industrial: aspectos teóricos e algumas evidências, com aplicação ao caso brasileiro. 1988. 171 f. Dissertação (Mestrado em Economia) - Universidade de Brasília, Brasília, DF, 1988. 
variáveis que mais explicaram esse tipo de comércio foram: tamanho de mercado, média das rendas per capita e desigualdade da renda per capita entre os países.

Machado e Markwald (1997) analisam em que medida a criação do Mercosul contribuiu para o incremento do comércio intraindustrial entre o Brasil e a Argentina durante o período de 1990 a 1996. A principal conclusão foi a de que a implementação do Mercosul intensificou as trocas intraindustriais bilaterais. Nessa mesma linha, Vasconcelos (2003) avaliou a contribuição do comércio intraindustrial para o crescimento do fluxo comercial brasileiro intramercosul no período 1990 a 1998.

Vasconcelos (2003) analisou o comércio intraindustrial entre o Brasil e o Mercosul no período compreendido entre 1990 e 1998 e, adicionalmente, verificou qual a importância desse padrão de comércio para o crescimento do comércio total. Nesse trabalho verificou-se que as trocas intraindustriais entre o Brasil e o Mercosul oscilaram entre 48\% a 64\% entre 1990 e 1998. Além disso, se observou que, no período compreendido entre 1990 e 1992, o crescimento do comércio intraindustrial representou $43 \%$ do crescimento do comércio total. Já nos períodos posteriores, de 1994 a 1996 e de 1996 a 1998, a contribuição do comércio intraindustrial foi de $78 \%$ e $89 \%$, respectivamente. Dessa forma, as evidências reportadas nesse trabalho sugerem que as trocas intraindustriais contribuíram de forma significativa para o crescimento do comércio total entre o Brasil e o Mercosul no período compreendido entre 1990 e 1998.

Baltar (2008) avaliou a inserção internacional do Brasil por meio da mensuração dos diferentes padrões de comércio: comércio interindustrial, comércio intraindustrial horizontal e comércio intraindustrial vertical, para os períodos de 1996 a 1998 e 2003 a 2005. Nesse caso, a autora verificou que o comércio intraindustrial apresentou elevação para os produtos diferenciados intensivos em PEBD e diferenciados com fornecedores especializados. No que tange à diferenciação dos produtos no comércio intraindustrial, encontrou-se evidências de que esse padrão de comércio ocorre, sobretudo, em produtos verticalmente diferenciados, em que os produtos exportados pelos Brasil possuem qualidade inferior a dos produtos importados dos seus parceiros comerciais.

Curzel, Montoro e Vartanian (2010) mensuram o comércio intraindustrial entre o Brasil e os países do Mercosul para o período entre 1996 e 2005. O estudo concentrou-se nas indústrias que possuem maior participação no intercâmbio bilateral. Nesse caso, os resultados obtidos demonstram que as indústrias de produtos plásticos e veículos são aquelas que possuem os maiores índices de trocas intraindustriais.

Um ponto importante a ressaltar em relação aos trabalhos anteriormente listados é que os mesmos não procuram analisar os determinantes do comércio intraindustrial brasileiro, sendo assim, na tentativa de preencher essa lacuna, o 
presente artigo tem como objetivo principal não apenas analisar o comércio intraindustrial (CII) entre o Brasil e os países da Organização para a Cooperação e Desenvolvimento Econômico (OCDE), no período compreendido entre 2000 a 2009, mas, especificamente, pretende-se decompor o comércio intraindustrial (CII) bilateral e analisar os seus determinantes. A hipótese central do presente estudo é a de que o comércio intraindustrial entre o Brasil e os países da OCDE possui natureza vertical, em que a qualidade dos produtos exportados pelo Brasil é inferior à qualidade dos produtos exportados pelos países da OCDE.

Os principais resultados encontrados mostram que os Estados Unidos e o México são os países da OCDE que possuem o maior volume e índice de comércio intraindustrial (CII) com o Brasil, respectivamente. Ademais, verificou-se que, em todas as relações bilaterais, o comércio intraindustrial vertical (CIIV) é superior ao comércio intraindustrial horizontal $(\mathrm{CIIH})$, em que os produtos exportados pelo Brasil possuem qualidade inferior a dos produtos importados. Após a decomposição do comércio intraindustrial (CII), partiu-se para a análise empírica dos seus determinantes. Nesse caso, testou-se a hipótese de que a diferença entre as dotações de fatores constitui-se um fator importante para a predominância do comércio intraindustrial vertical. A estimação do modelo empírico foi realizada por meio da técnica econométrica de dados em painel. Conforme os resultados obtidos do método de efeitos aleatórios, verificou-se que, a 10\% de significância, a diferença na dotação de fatores tem um efeito positivo sobre o comércio intraindustrial vertical, o que corrobora a hipótese central do modelo teórico de Falvey e Kierzkowski (1987).

Além da presente introdução, este estudo possui outras quatro seções. $\mathrm{Na}$ seção 2, mensura-se e decompõe-se o comércio intraindustrial (CII) entre o Brasil e os países da OCDE. Na seção 3, descreve-se a abordagem empírica. Na seção 4, tem-se a análise dos resultados obtidos. Por fim, na seção 5, têm-se as considerações finais do trabalho.

\section{Referencial Teórico}

O modelo teórico que balizará o presente artigo será o desenvolvido por Falvey e Kierzkowski (1987). Nesse modelo, para derivar o padrão de comércio intraindustrial (CII), os autores utilizam uma estrutura $2 \times 2 \times 2$, isto é, dois países, $\mathrm{N}$ (Norte) e S (Sul), dois fatores de produção, capital (K) e trabalho (L), e dois bens, um homogêneo e outro diferenciado, sendo que a qualidade do bem diferenciado é determinada num espaço contínuo compreendido no intervalo $\left[0, s_{\max }\right]$.

O fator trabalho (L) é utilizado tanto na produção do bem homogêneo quanto na produção do bem diferenciado, ao passo que o fator capital (K) é utilizado apenas na produção do bem diferenciado. A produção de uma unidade do bem 
homogêneo requer $\mathrm{b}$ unidades de trabalho, enquanto que a produção de uma unidade do bem diferenciado, de qualidade s, requer uma unidade de trabalho e $s$ unidades de capital. Assim, a qualidade do bem diferenciado será diretamente proporcional à quantidade de capital empregada na sua produção.

Assumindo uma estrutura de mercado que opera em concorrência perfeita, e considerando o bem homogêneo como numerário, o preço do bem diferenciado de qualidade $s$ será fixado como $p(s)=\omega+s r$, em que $\omega$ será o custo com salário e $r$ a rentabilidade do capital. Considera-se, ainda, que os países apresentam diferenças nas dotações de fatores, em termos da razão capital/trabalho. Nessa situação, assumindo que o país do Norte $(\mathrm{N})$ possui uma razão capital/trabalho superior à do país do Sul (S), ocorrerá $b_{N}<b_{s}$, implicando em $\omega_{\mathrm{N}}>\omega_{\mathrm{S}}$, caso os países produzam o bem homogêneo com livre comércio. Desse modo, para que ocorra o comércio intraindustrial, será necessário que a rentabilidade do capital seja menor em $\mathrm{N}$ do que em S, ou seja, $r_{N}<r_{S}$. Assim, caso $C_{\mathrm{N}}(\mathrm{s})=\omega_{\mathrm{N}}+\mathrm{sr}_{\mathrm{N}}$ e $\mathrm{C}_{\mathrm{S}}(\mathrm{s})=\omega_{\mathrm{S}}+\mathrm{sr}_{\mathrm{S}}$ sejam, respectivamente, os custos de produção do produto de qualidade $s$ em $\mathrm{Ne}$ em S, existirá uma qualidade marginal $s_{m}$, tal que:

$$
p(s)= \begin{cases}C_{A}(s) \leq C_{B}(s) \text { se } & S \geq S_{m} \\ C_{A}(s) \geq C_{B}(s) \text { se } & S \leq S_{m}\end{cases}
$$

Nesse caso, como o país $\mathrm{N}$ possui abundância em capital físico, o mesmo terá menor custo de produção para as variedades de qualidade superior a $s_{m}$, isto é, terá vantagens comparativas na produção das variedades de alta qualidade. Já o país S, como é escasso em capital, terá menor custo de produção para as variedades de qualidade inferior a $s_{m}$, em outras palavras, esse país terá vantagens comparativas na produção das variedades de baixa qualidade.

Para garantir que exista o comércio intraindustrial, considera-se que a renda seja distribuída de maneira desigual tanto em N quanto em S. Dessa forma, os indivíduos de baixa renda, independente do seu país de origem, demandarão os produtos de baixa qualidade, produzidos em $\mathrm{S}$, ao passo que os indivíduos de renda mais alta demandarão os produtos de alta qualidade, produzidos em $\mathrm{N}$.

Seguindo a intuição do modelo teórico de Falvey e Kierzkowski (1987), testa-se a seguinte hipótese: devido às diferenças nas razões capital/trabalho, o comércio intraindustrial bilateral entre o Brasil e os países da OCDE ocorre, majoritariamente, em produtos verticalmente diferenciados, e como o Brasil possui uma relação capital/trabalho inferior à maioria dos países da OCDE, a qualidade dos produtos exportados pelo Brasil tende a ser inferior à qualidade dos produtos exportados pelos países da OCDE, na maior parte dos casos. 


\section{Método de Mensuração e Decomposição do Comércio Intraindustrial}

Para mensurar e decompor o comércio intraindustrial bilateral (CII), utiliza-se a mesma metodologia de Greenaway, Hine e Milner (1994). Nesse caso, assumindo que o volume comercial total ( $T T_{k i j l}$ ), entre os países $i$ e $j$, no ano $t$, é igual ao somátório do comércio interindústria $\left(C E I_{k i j t}\right)$ com o comércio intraindústria $\left(C I I_{k j i l}\right)$, observa-se para o produto $k$ que:

$$
T T_{k i j t}=C E I_{k i j t}+C I I_{k i j t}
$$

Onde:

$$
\begin{gathered}
T T_{k i j t}=\left(X_{k i j t}+M_{k i j t}\right) \\
C E I_{k i j t}=\left|X_{k i j t}-M_{k i j t}\right|
\end{gathered}
$$

Logo, a partir das equações 2 e 3, nota-se que o comércio intraindustrial $\left(C I I_{k i j l}\right)$ pode ser obtido do seguinte modo:

$$
C I I_{k i j t}=\left(X_{k i j t}+M_{k i j t}\right)-\left|X_{k i j t}-M_{k i j t}\right|
$$

Ressalta-se que $X_{k i j t}$ e $M_{k i j t}$ denotam, respectivamente, o valor monetário das exportações e das importações do produto $k$, comercializado entre os países $i$ e $j$, no ano $t$.

Para transformar o volume de comércio intraindustrial $\left(C I I_{k i j}\right)$ em forma de índice, deve-se dividir a equação 4 pela equação 2 , o que resultará em:

$$
G L_{k i j t}=1-\frac{\left|X_{k i j t}-M_{k i j t}\right|}{\left(X_{k i j t}+M_{k i j t}\right)}
$$

A equação 5 denota o índice de Grubel e Lloyd (1975) (doravante índice GL). Cabe ressaltar que o índice GL varia no intervalo $(0,1)$, sendo que, quanto mais o mesmo se aproximar da unidade, maior será a participação do comércio intraindustrial (CII) no comércio total.

Após a mensuração do comércio intraindustrial (CII), deve-se defini-lo como horizontal (CIIH) ou vertical (CIIV), nesse caso, isso será feito conforme o critério de similaridade do produto (CRESPO; FONTOURA, 2004). Esse critério define o padrão de diferenciação dos produtos a partir da razão $(\lambda)$ obtida da divisão do va- 
lor unitário do produto $(k)$ exportado $\left(V U X_{k i j l}\right)$ e importado $\left(V U M_{k i j l}\right)$ entre os países i e j, no ano $t$, ou seja: $\lambda=V U X_{k i j l} / V U M_{k j i t}{ }^{2}$

Nesse caso, quando a razão entre os valores unitários $(\lambda)$ se mantém próxima da unidade, considera-se que os produtos comercializados não possuem diferenças significativas entre si, em consequência disso, os mesmos serão definidos como horizontalmente diferenciados, ou seja, o comércio intraindustrial será horizontal $(\mathrm{CIIH})$. Caso contrário, quando a razão entre os valores unitários afasta-se demasiadamente da unidade, considera-se que os produtos comercializados são verticalmente diferenciados, ou seja, nessa situação, o comércio intraindustrial será vertical (CIIV).

Cabe ressaltar que o critério utilizado para definir se $\lambda$ está próximo ou afastado da unidade será baseado no intervalo de dispersão, definido por: $[(1-\alpha) ;(1+\alpha)]$. Assim, quando $\lambda$ pertencer a esse intervalo, considera-se que os produtos comercializados são horizontalmente diferenciados, caso contrário, serão definidos como verticalmente diferenciados. No presente trabalho, seguindo Greenaway, Hine e Milner (1994) e Fontagné e Freudenberg (1997), serão utilizados dois fatores de dispersão, $\alpha=15 \%$ e $\alpha=25 \%$. Sendo assim, serão obtidos dois intervalos assim definidos: $[0,85 ; 1,15]$ e $[0,75 ; 1,25]$, para $\alpha=15 \%$ e $\alpha=25 \%$, respectivamente.

Destaca-se, ainda, que nos casos em que os produtos forem verticalmente diferenciados, pode-se definir o CIIV, sob o ponto de vista do país exportador, como vertical inferior (CIIVINF) ou como vertical superior (CIIV SUP), quando $\lambda<(1-\alpha)$ ou $\lambda>(1+\alpha)$, respectivamente. No primeiro caso, os produtos do país exportador possuem qualidade inferior à dos produtos importados, e o contrário ocorre na segunda situação.

É importante ressaltar que o critério de similaridade acima descrito parte do pressuposto de que o valor unitário do produto serve de proxy para a sua qualidade. Conforme Greenaway, Hine e Milner (1994), a justificativa para a utilização do preço como proxy para a qualidade deriva-se da pressuposição de que os consumidores possuem informação perfeita e são capazes de distinguir a qualidade dos produtos ofertados, em que o bem de maior qualidade tenha um valor unitário superior ao do bem de pior qualidade. Já Stiglitz (1987) considera que o valor unitário refletirá a qualidade do produto até mesmo quando o consumidor não possuir informação perfeita.

Fontagné, Gaulier e Zignago (2007) reconhecem que a associação direta entre o valor unitário e a qualidade apresenta algumas limitações, pois outros fatores, como, por exemplo, as estruturas de mercado, as diferenças de custos e as dife-

$2 \quad$ Seguindo Greenaway, Hine e Milner (1994), Fontgné e Freudenberg (1997) e Schott (2004), o valor unitário do produto será calculado por meio da divisão do valor monetário pela quantidade comercializada, ou seja: $V U=V / Q$. Conforme Gullstrand (2000), a utilização do valor unitário como proxy da qualidade necessita da suposição de que não existe correlação entre a qualidade e o peso do produto, pois, se isso ocorrer, o cálculo do valor unitário será viesado. 
renças de tecnologia, podem afetar o valor unitário do produto e, com isso, levar a uma interpretação errônea sobre a qualidade. Porém, os autores argumentam que como esses outros fatores são extremamente difíceis de mensurar, torna-se plausível assumir que o valor unitário do produto seja utilizado como uma proxy da qualidade dos produtos.

Para mensurar e decompor o comércio intraindustrial (CII) entre o Brasil e os países da OCDE, utilizar-se-á a base de dados da Baci. Ressalta-se que a Baci reporta os dados bilaterais de comércio, na classificação do sistema harmonizado (harmonized system), no nível de desagregação de seis dígitos. ${ }^{3}$ Destaca-se que o nível de desagregação utilizado no presente trabalho é bem mais elevado do que o dos trabalhos precedentes que, em geral, utilizam dados de comércio ao nível de três dígitos, como em Vasconcelos (2003). ${ }^{4}$

\section{Caracterização do Comércio Intraindustrial entre o Brasil e a OCDE}

Na presente seção, realiza-se uma caracterização do comércio intraindustrial entre o Brasil e os países da OCDE. ${ }^{5}$

A Tabela 1 reporta o volume do comércio intraindustrial (CII) bilateral, média para o período 2000-2009, entre o Brasil e os países da OCDE. Nota-se que o volume total de comércio intraindustrial (CII) ficou pouco abaixo de US $\$ 13$ bilhões, sendo que os volumes de comércio intraindustrial horizontal (CIIH) e vertical (CIIV) se alteram levemente, dependendo do nível de dispersão adotado ( $\alpha$ $=15 \%$ ou $\alpha=25 \%$ ), mas em ambas as situações o CIIV é superior ao $\mathrm{CIIH}$, em que, dependendo do fator de dispersão adotado, o CIIV representa de $73,4 \%$ a $83,8 \%$ do comércio intraindustrial (CII). No que tange às relações bilaterais, nota-se que os países que possuem maiores volumes de comércio intraindustrial (CII) com o Brasil são: Estados Unidos (US\$ 6.309), Alemanha (US\$ 1.971) e México (US\$ 1.030). Ressalta-se, ainda, que, em todas as relações bilaterais, o CIIV é superior ao $\mathrm{CIIH}$, e que esse resultado se mantém independentemente do fator de dispersão utilizado.

Dentre as razões apontadas para um maior comércio intraindustrial (CII), pode-se citar a maior globalização a partir da década dos 1990, além do maior

\footnotetext{
3 A Baci é publicada pela instituição de pesquisa Cepii, e está disponível para download em: <http:// www.cepii.fr/anglaisgraph/bdd/baci.htm > . Para mais detalhes da base Baci, ver Gaulier e Zignago (2010).

$4 \quad$ A mensuração do CII com dados mais agregados tende a superestimar a magnitude do índice GL. Finger (1975) chama isso de "viés categórico". Dessa forma, a utilização de dados mais desagregados tende a mitigar esse "viés categórico" e tornar os resultados mais confiáveis.

5 Para mensurar e decompor o CII, foram utilizadas 674.087 observações, sendo que 9.611 (1,43\%) tiveram que ser excluídas por não informarem a quantidade comercializada.
} 
crescimento de políticas de liberalização comercial em boa parte dos países em desenvolvimento (FONTAGNÉ; FREUDENBERG; GAULIER, 2006).

Tabela 1 - Volume de comércio intraindustrial (CII), intraindustrial horizontal $(\mathrm{CIIH})$ e intraindustrial vertical (CIIV) entre o Brasil e os países da OCDE média para 2000/2009 (em milhões de US\$)

\begin{tabular}{|c|c|c|c|c|c|}
\hline \multirow{2}{*}{ País } & \multirow{2}{*}{ CII } & \multicolumn{2}{|c|}{$\alpha=15 \%$} & \multicolumn{2}{|c|}{$\alpha=25 \%$} \\
\hline & & CII-H & CII-V & CII-H & CII-V \\
\hline Alemanha & $1.971,0$ & 534,8 & $1.436,2$ & 804,2 & $1.166,8$ \\
\hline Austrália & 34,8 & 6,3 & 28,5 & 10,1 & 24,7 \\
\hline Áustria & 39,5 & 5,3 & 34,2 & 8,9 & 30,6 \\
\hline $\begin{array}{c}\text { Bélgica- } \\
\text {-Luxemburgo }\end{array}$ & 217,0 & 31,5 & 185,5 & 59,5 & 157,5 \\
\hline Canadá & 187,8 & 29,6 & 158,2 & 47,3 & 140,5 \\
\hline Coreia do Sul & 90,1 & 24,0 & 66,1 & 29,9 & 60,1 \\
\hline Dinamarca & 45,2 & 1,6 & 43,6 & 4,6 & 40,6 \\
\hline Eslováquia & 3,9 & 0,3 & 3,7 & 0,7 & 3,2 \\
\hline Espanha & 288,1 & 35,3 & 252,8 & 65,1 & 223,0 \\
\hline Estados Unidos & $6.309,6$ & 972,0 & $5.337,6$ & $1.548,3$ & $4.761,3$ \\
\hline Finlândia & 35,6 & 3,3 & 32,4 & 5,1 & 30,5 \\
\hline França & 701,4 & 85,1 & 616,3 & 175,7 & 525,7 \\
\hline Grécia & 3,9 & 0,4 & 3,5 & 0,6 & 3,3 \\
\hline Holanda & 258,5 & 26,5 & 232,0 & 40,5 & 218,0 \\
\hline Hungria & 20,1 & 1,9 & 18,2 & 2,8 & 17,3 \\
\hline Irlanda & 23,2 & 2,9 & 20,3 & 4,2 & 19,0 \\
\hline Islândia & 0,0 & 0,0 & 0,0 & 0,0 & 0,0 \\
\hline Itália & 622,3 & 77,8 & 544,5 & 133,9 & 488,5 \\
\hline Japão & 210,0 & 25,9 & 184,1 & 43,3 & 166,7 \\
\hline México & $1.030,8$ & 133,7 & 897,1 & 293,6 & 737,2 \\
\hline Noruega & 18,0 & 1,8 & 16,2 & 2,8 & 15,2 \\
\hline Nova Zelândia & 3,2 & 0,6 & 2,6 & 0,8 & 2,4 \\
\hline Polônia & 28,6 & 2,9 & 25,7 & 5,8 & 22,7 \\
\hline Portugal & 67,3 & 4,8 & 62,5 & 7,9 & 59,3 \\
\hline Reino Unido & 394,5 & 46,0 & 348,6 & 72,7 & 321,8 \\
\hline $\begin{array}{l}\text { República } \\
\text { Tcheca }\end{array}$ & 21,9 & 2,1 & 19,7 & 3,5 & 18,4 \\
\hline Suécia & 206,6 & 27,4 & 179,2 & 49,9 & 156,7 \\
\hline Suíça & 70,3 & 7,7 & 62,6 & 12,2 & 58,1 \\
\hline Turquia & 45,7 & 4,0 & 41,7 & 6,3 & 39,4 \\
\hline Total & $12.948,8$ & $2.095,7$ & $10.853,1$ & 3.440 .1 & 9.508 .6 \\
\hline
\end{tabular}

Fonte: Elaboração própria a partir de cálculo dos autores. 
Na Tabela 2, apresenta-se o comércio intraindustrial (CII) entre o Brasil e os países da OCDE em forma de índice. Nesse caso, verifica-se que o índice GL ficou em torno de 0,12 , sendo que para o comércio intraindustrial horizontal (GL-H) e intraindustrial vertical (GL-V) os índices foram, respectivamente, de 0,02 e 0,10, para $\alpha=15 \%$, e de 0,03 e 0,09 para $\alpha=25 \%$. Observa-se que os países que possuem trocas intraindustriais mais intensas com o Brasil são: México $(0,21)$, Estados Unidos $(0,18)$ e Alemanha $(0,16)$. Nesse caso, evidenciou-se, na Tabela 1 , que o México é apenas o terceiro principal parceiro comercial em termos de volume de comércio intraindustrial, mas é o principal parceiro comercial do Brasil quando se analisa as trocas intraindustriais em forma de índice. 
Tabela 2 - Índice de comércio intraindustrial (GL), intraindustrial horizontal (GL-H) e intraindustrial vertical (GL-V) entre o Brasil e os países da OCDE, em termos bilaterais (média para 2000/2009)

\begin{tabular}{|c|c|c|c|c|c|}
\hline \multirow{2}{*}{ País } & \multirow{2}{*}{ GL } & \multicolumn{2}{|c|}{$\alpha=15 \%$} & \multicolumn{2}{|c|}{$\alpha=25 \%$} \\
\hline & & GL-H & GL-V & GL-H & GL-V \\
\hline Alemanha & 0,15 & 0,04 & 0,11 & 0,06 & 0,09 \\
\hline Austrália & 0,03 & 0,01 & 0,03 & 0,01 & 0,02 \\
\hline Áustria & 0,05 & 0,01 & 0,04 & 0,01 & 0,04 \\
\hline $\begin{array}{c}\text { Bélgica- } \\
\text {-Luxemburgo }\end{array}$ & 0,06 & 0,01 & 0,05 & 0,02 & 0,04 \\
\hline Canadá & 0,06 & 0,01 & 0,05 & 0,02 & 0,05 \\
\hline Coreia do Sul & 0,02 & 0,01 & 0,01 & 0,01 & 0,01 \\
\hline Dinamarca & 0,09 & 0,00 & 0,09 & 0,01 & 0,08 \\
\hline Eslováquia & 0,03 & 0,00 & 0,03 & 0,00 & 0,03 \\
\hline Espanha & 0,08 & 0,01 & 0,07 & 0,02 & 0,06 \\
\hline Estados Unidos & 0,18 & 0,03 & 0,15 & 0,04 & 0,13 \\
\hline Finlândia & 0,04 & 0,00 & 0,03 & 0,01 & 0,03 \\
\hline França & 0,12 & 0,01 & 0,11 & 0,03 & 0,09 \\
\hline Grécia & 0,01 & 0,00 & 0,01 & 0,00 & 0,01 \\
\hline Holanda & 0,04 & 0,00 & 0,04 & 0,01 & 0,03 \\
\hline Hungria & 0,08 & 0,01 & 0,07 & 0,01 & 0,07 \\
\hline Irlanda & 0,04 & 0,00 & 0,03 & 0,01 & 0,03 \\
\hline Islândia & 0,00 & 0,00 & 0,00 & 0,00 & 0,00 \\
\hline Itália & 0,10 & 0,01 & 0,09 & 0,02 & 0,08 \\
\hline Japão & 0,03 & 0,00 & 0,03 & 0,01 & 0,02 \\
\hline México & 0,21 & 0,03 & 0,19 & 0,06 & 0,15 \\
\hline Noruega & 0,02 & 0,00 & 0,02 & 0,00 & 0,02 \\
\hline $\begin{array}{c}\text { Nova } \\
\text { Zelândia }\end{array}$ & 0,03 & 0,00 & 0,03 & 0,01 & 0,02 \\
\hline Polônia & 0,04 & 0,00 & 0,04 & 0,01 & 0,03 \\
\hline Portugal & 0,05 & 0,00 & 0,05 & 0,01 & 0,04 \\
\hline Reino Unido & 0,09 & 0,01 & 0,08 & 0,02 & 0,07 \\
\hline $\begin{array}{l}\text { República } \\
\text { Tcheca }\end{array}$ & 0,06 & 0,01 & 0,05 & 0,01 & 0,05 \\
\hline Suécia & 0,15 & 0,02 & 0,13 & 0,04 & 0,11 \\
\hline Suíça & 0,03 & 0,00 & 0,03 & 0,01 & 0,02 \\
\hline Turquia & 0,06 & 0,00 & 0,06 & 0,01 & 0,05 \\
\hline Total & 0,12 & 0,02 & 0,10 & 0,03 & 0,09 \\
\hline
\end{tabular}

Fonte: Elaboração própria a partir de cálculo dos autores. 
Como a maior parte do comércio intraindustrial (CII) entre o Brasil e os países da OCDE ocorre, sobretudo, em produtos verticalmente diferenciados, é interessante verificar se a qualidade dos produtos exportados pelo Brasil é superior ou inferior à dos produtos importados. Desse modo, decompõe-se o volume de comércio intraindustrial vertical (CIIV) em vertical inferior (CIIV ${ }^{\mathrm{NF}}$ ) e vertical superior (CIIVSUP); cujos resultados estão reportados na Tabela 3.

Nota-se na Tabela 3 que na maior parte dos casos os produtos exportados pelo Brasil possuem qualidade inferior a dos produtos importados dos países da OCDE, pois o CIIV ${ }^{\mathrm{iNF}}$ supera o CIIVSup. Cabe também destacar que esses resultados são robustos, pois não se alteram com a mudança no fator de dispersão ( $\alpha$ ). Chama a atenção o fato do CIIV SUP superar o CIIV ${ }^{I N F}$ nos seguintes casos: Coreia do Sul, Finlândia, Hungria, Republica Tcheca, Irlanda e Turquia. Contudo, uma análise mais detalhada de cada uma dessas situações está fora do escopo do presente trabalho. 
Tabela 3 - Decomposição do comércio intraindustrial vertical (CIIV) em vertical inferior (CIIV ${ }^{\mathrm{INF}}$ ) e vertical superior (CIIV ${ }^{\mathrm{SUP}}$ ) (em porcentagem)

\begin{tabular}{|c|c|c|c|c|}
\hline \multirow{2}{*}{ País } & \multicolumn{2}{|c|}{$\alpha=15 \%$} & \multicolumn{2}{|c|}{$\alpha=25 \%$} \\
\hline & CIIVINF $^{\text {INF }}$ & CIIVSUP & CIIVINF $^{\text {INF }}$ & CIIVSUP \\
\hline Alemanha & 61,6 & 38,4 & 60,7 & 39,3 \\
\hline Austrália & 63,1 & 36,9 & 65,5 & 34,5 \\
\hline Áustria & 54,5 & 45,5 & 53,4 & 46,6 \\
\hline $\begin{array}{c}\text { Bélgica- } \\
\text {-Luxemburgo }\end{array}$ & 68,3 & 31,7 & 67,7 & 32,3 \\
\hline Canadá & 57,0 & 43,0 & 57,7 & 42,3 \\
\hline Coreia do Sul & 34,3 & 65,7 & 31,4 & 68,6 \\
\hline Dinamarca & 67,0 & 33,0 & 65,8 & 34,2 \\
\hline Eslováquia & 52,0 & 48,0 & 48,5 & 51,5 \\
\hline Espanha & 52,6 & 47,4 & 54,1 & 45,9 \\
\hline Estados Unidos & 69,0 & 31,0 & 69,3 & 30,7 \\
\hline Finlândia & 39,8 & 60,2 & 37,0 & 63,0 \\
\hline França & 62,3 & 37,7 & 60,5 & 39,5 \\
\hline Grécia & 57,8 & 42,2 & 55,9 & 44,1 \\
\hline Holanda & 74,9 & 25,1 & 75,3 & 24,7 \\
\hline Hungria & 30,4 & 69,6 & 28,8 & 71,2 \\
\hline Irlanda & 21,7 & 78,3 & 20,1 & 79,9 \\
\hline Islândia & 60,1 & 39,9 & 60,1 & 39,9 \\
\hline Itália & 69,8 & 30,2 & 71,0 & 29,0 \\
\hline Japão & 73,1 & 26,9 & 72,9 & 27,1 \\
\hline México & 68,5 & 31,5 & 65,9 & 34,1 \\
\hline Noruega & 57,3 & 42,7 & 56,2 & 43,8 \\
\hline Nova Zelândia & 83,7 & 21,9 & 78,5 & 21,5 \\
\hline Polônia & 58,4 & 41,6 & 56,6 & 43,4 \\
\hline Portugal & 68,9 & 31,1 & 68,9 & 31,1 \\
\hline Reino Unido & 73,8 & 26,2 & 73,8 & 26,2 \\
\hline $\begin{array}{l}\text { República } \\
\text { Tcheca }\end{array}$ & 41,6 & 58,4 & 40,0 & 60,0 \\
\hline Suécia & 66,2 & 33,8 & 63,0 & 37,0 \\
\hline Suíça & 58,6 & 41,4 & 58,0 & 42,0 \\
\hline Turquia & 34,3 & 65,7 & 32,9 & 67,1 \\
\hline Total & 67,2 & 33,0 & 66,3 & 33,7 \\
\hline
\end{tabular}

Fonte: Elaboração própria a partir de cálculo dos autores. 
Em síntese, os resultados apresentados nesta seção sugerem que existe uma diferenciação intraproduto, na qual o Brasil tende a ser o exportador líquido das variedades de baixa qualidade e importador líquido das variedades de alta qualidade. Dessa forma, os dados até aqui analisados sugerem que o comércio intraindustrial entre o Brasil e a OCDE é determinado pelas vantagens comparativas dos países, como sugere o modelo teórico de Falvey e Kierzkowski (1987), apresentado na seção 2 do presente estudo.

\section{Abordagem Empírica}

Na seção anterior, caracterizou-se que o comércio intraindustrial entre o Brasil e a OCDE ocorre, predominantemente, em produtos verticalmente diferenciados. Desse modo, na presente seção, seguindo a intuição do modelo teórico de Falvey e Kierzkowski (1987), será testado se a diferença entre as dotações de fatores se constitui em um fator determinante do comércio intraindustrial vertical (CIIV).

\subsection{Modelo Econométrico}

Para testar se a diferença entre as dotações de fatores constitui um determinante do comércio intraindustrial vertical (CIIV), será formulado um modelo empírico baseado na equação gravitacional de comércio. A equação gravitacional estabelece que os fluxos bilaterais de comércio são positivamente relacionados ao tamanho econômico dos países e inversamente à distância geográfica. Especificamente, na forma logaritmizada, o modelo empírico que será estimado possui a seguinte forma. ${ }^{6}$

$$
\ln C I I V_{i j t}=\alpha_{0}+\alpha_{t}+\beta_{1} \operatorname{lnPIB}_{i j t}+\beta_{2} \ln D I S T_{i j}+\beta_{3} \operatorname{lnDIF}_{i j t}+\theta_{1 j}+u_{i j t}
$$

Onde: $C I I V_{i j t}$ é o volume de comércio intraindustrial vertical entre o Brasil e o país $j$, no ano $t ; P_{i j}$ denota a média aritmética do PIB do Brasil e do país $j$, no ano $t$; $D I S T_{i j}$ denota a distância geográfica entre o Brasil e o país $j$; $D I F_{i j t}$ é a diferença na dotação de fatores do Brasil e do país $j$, no ano $t ; \alpha_{0}$ é o intercepto, comum para todos os anos, e para todas as relações bilaterais de comércio; $\alpha_{t}$ é o intercepto que varia com o tempo, mas é comum para todas as relações bilaterais de comércio; $\theta_{i j}$ é a heterogeneidade não observada presente nas relações bilaterais de comércio, que é invariante no tempo; e $u_{i j t}$ corresponde ao termo de erro idiossincrático.

\footnotetext{
$\overline{6}$ O modelo empírico utilizado é semelhante ao utilizado por Emirhan (2005).
} 
Alguns trabalhos encontrados na literatura (EKANAYAKE et al., 2007; JANSEN; LÜTHJE, 2009) utilizam como variável dependente o índice ao invés do volume de comércio intraindustrial. Contudo, Nilsson (1999) questiona a utilização do índice GL como variável dependente pelo fato de não possuir escala. Nesse caso, é possível que uma relação bilateral de comércio tenha um alto índice de comércio intraindustrial, mas um baixo volume desse padrão de comércio. No presente estudo, isso realmente ocorre, pois, como verificado na seção 4, o México é o país que possui o maior índice de trocas intraindustriais com o Brasil, mas são os Estados Unidos que possuem o maior volume desse padrão de comércio. Dessa forma, seguindo Baleix e Egidio (2005) e Emirhan (2005), o modelo econométrico terá como variável dependente o volume de comércio intraindustrial bilateral (CIIV). Destaca-se que essa variável foi mensurada conforme a metodologia descrita na seção 3.

A variável PIB será utilizada como proxy para o tamanho do mercado consumidor. Greenaway e Tortensson (1997) destacam que a produção de produtos verticalmente diferenciados envolve altos custos fixos, então, dessa forma, o tamanho do mercado consumidor tem um papel relevante para a produção e comercialização desse tipo de produto. Vários trabalhos encontrados na literatura (CRESPO; FONTOURA, 2004; BALEIX; EGIDIO, 2005; JANSEN; LÜTHJE, 2009) encontram evidências robustas de que tamanho do mercado tem um impacto positivo sobre o comércio intraindustrial vertical, sendo assim, espera-se que $\beta_{1}>0$.

A distância geográfica (DIST) é utilizada como proxy para o custo de transporte (NILSSON, 1999). Ademais, é cabível salientar que o comércio de produtos diferenciados, que possuem maiores índices de comércio intraindustrial, tende a ser mais sensivel à distância geográfica do que o comércio de bens homogêneos. Conforme Rauch (1996), os produtos homogêneos são comercializados em mercados organizados, nos quais o preço e as características dos produtos são conhecidos por todas as partes envolvidas na negociação. Já a maior parte dos produtos diferenciados não é comercializada em mercados organizados. Nesse caso, tanto o preço quanto as características dos bens não são completamente conhecidos por todos os agentes envolvidos na negociação, isto é, o comércio de bens manufaturados envolve maiores assimetrias de informação. Diversos trabalhos encontrados na literatura apontam que a distância geográfica tem um impacto negativo sobre o comércio intraindustrial vertical (DURKIN; KRYGIER, 2000; CRESPO; FONTOURA, 2004; BALEIX; EGIDIO, 2005; EKANAYAKE et al., 2007; JANSEN; LÜTHJE, 2009), sendo assim, espera-se que $\beta_{2}<0$.

Conforme o modelo teórico de Falvey e Kierzkowski (1987), descrito na seção 2 do presente estudo, o comércio intraindustrial ocorre, principalmente, entre parceiros comerciais que possuem distintas dotações de fatores em termos de razão capital/trabalho. Nesse modelo, as nações mais desenvovidas, por possuírem 
maior razão capital/trabalho, devem se especializar na produção e comercialização das variedades de alta qualidade, ao passo que as nações menos desenvolvidas, como possuem menor razão capital/trabalho, se especializam na produção e comercialização das variedades de baixa qualidade. Dessa forma, a variável DIF inserida no modelo empírico descrito na equação (6) tem a propriedade de identificar a dissimilaridade entre as dotações de fatores dos parceiros comerciais. Helpman (1987) considera que a renda per capita pode ser utilizada como proxy para a dotação de fatores dos países, devido à correlação positiva existente entre a razão capital/trabalho e a renda per capita. ${ }^{7}$ Desse modo, seguindo esse raciocínio, utilizar-se-á a diferença absoluta no PIB per capita como proxy para a dissimilaridade entre as dotações de fatores (DIF). Seguindo o modelo teórico de Falvey e Kierzkowski (1987), espera-se que $\beta_{3}>0$.

Destaca-se, ainda, que o Apêndice A reporta algumas estatísticas descritivas das variáveis que integram o modelo econométrico especificado na equação $6 .^{8}$

\subsection{Estratégia de Estimação}

O banco de dados utilizado neste artigo possui 290 observações, referentes a 29 relações bilaterais de comércio entre o Brasil e os países da OCDE, observadas no período compreendido entre 2000 e 2009. Sendo assim, como a base de dados reúne tanto observações de cross-section quanto de série de tempo, a estimação do modelo descrito na equação 6 será feita por meio da técnica de dados em painel.

A principal motivação para a utilização da técnica de dados em painel é a possibilidade do controle da heterogeneidade não observada $\left(\theta_{i j}\right)$, que está presente nas relações bilaterais de comércio. ${ }^{9}$ Nessa situação, conforme Wooldridge (2002), caso a heterogeneidade não observada $\left(\theta_{i j}\right)$ não seja correlacionada com os regressores da equação, o método de mínimos quadrados ordinários (MQO) pooled fornece estimativas não viesadas e consistentes dos parâmetros. Entretanto, estimativas mais eficientes podem ser obtidas por meio do método de efeitos aleatórios (random effects - RE). Caso a heterogeneidade não observada $\left(\theta_{i j}\right)$ seja corre-

$7 \quad$ A lógica para a utilização do PIB per capita como proxy da razão capital/trabalho é a seguinte: considere que $Y=F(K, L)$, em que $Y$ denota o PIB, $K$ é a quantidade de capital físico, $L$ denota o número de trabalhadores e $F$ (.) é uma função homogênea de grau um. Dessa forma, dividindo ambos os lados da função por $L$, temos: $y=f(k)$; onde $y=Y / L$ e $k=K / L$.

8 As variáveis utilizadas no presente trabalho são oriundas de diferentes fontes: os dados de comércio são oriundos da base Baci; o PIB e o PIB per capita estão mensurados em termos da paridade de poder de compra (PPC) e ambos foram obtidos junto à base do FMI. Por fim, a distância geográfica foi obtida junto à base da Cepii.

$9 \quad \quad$ Nas palavras de Cheng e Wall (2005): "With such heterogeneity, a country would export different amounts to two countries, even though the two export markets have the same GDPs and are equidistant from the exporter. This can be because there are historical, cultural, ethnic, political, or geographic factors that affect the level of trade and are correlated with the gravity variables". 
lacionada com os regressores da equação, tanto os parâmetros obtidos a partir do método $\mathrm{MQO}$ pooled quanto os obtidos por efeitos aleatórios (RE) serão viesados e inconsistentes. Nessa situação, os parâmetros podem ser estimados de maneira não viesada e consistente por meio de efeitos fixos (fixed effect - FE).

\subsection{Análise dos Resultados}

As estimações dos parâmetros estão reportadas na Tabela 4. Primeiramente, cabe ressaltar que o teste de Breusch-Pagan, aplicado ao modelo de efeitos aleatórios $(\mathrm{RE})$, detectou a presença da heterogeneidade não observada nas estimações $\left(\theta_{i j}\right)$. Além disso, por meio do teste de Hausman, verificou-se que a heterogeneidade não observada $\left(\theta_{i j}\right)$ possui um comportamento aleatório, sendo assim, os resultados obtidos a partir do modelo com efeitos aleatórios (RE) são preferíveis em relação aos obtidos a partir do modelo com efeitos fixos (FE). Desse modo, o resultado obtido a partir do método de efeitos aleatórios (RE) foi usado como benchmark. Para superar os problemas de heterocedasticidade e de autocorrelação, todas as estimações foram realizadas com variância robusta do tipo Huber/White/ Sandwich (HUBER, 1967).

De maneira geral, destaca-se que os modelos estimados apresentaram um bom ajuste aos dados, tendo em vista que o coeficiente de determinação (R2) variou de 0,43 a 0,51. Em todas as estimações, os parâmetros apresentaram os sinais esperados, mas o nível de significância se altera profundamente entre os métodos utilizados. Na coluna (I) estão reportados os resultados obtidos do método MQO pooled. Nota-se que a variável PIB e a distância geográfica (DIST) possuem níveis de significância de $1 \%$ e 5\%, respectivamente. Já a variável DIF não foi estatisticamente significativa. Na coluna (II) estão os resultados obtidos do método de efeitos aleatórios (RE). Nesse caso, observa-se que as variáveis PIB e DIST, assim como no modelo MQO pooled, apresentaram níveis de significância de 1\% e 5\%, respectivamente. Já a variável DIF foi significativa a 10\%. Na coluna (III) estão reportados os resultados obtidos do método de efeitos fixos (FE). Nesse modelo, apenas a variável PIB apresentou significância estatística (10\%).

Em síntese, conforme as estimativas obtidas a partir do modelo de efeitos aleatórios (RE), verifica-se que as variáveis PIB e DIST têm, respectivamente, impactos positivo e negativo sobre o volume de comércio intraindustrial vertical bilateral, ou seja, o Brasil possui, em média, maiores volumes de comércio intraindustrial vertical com os parceiros comerciais com maior tamanho econômico e geograficamente mais próximos. Ademais, os resultados sugerem que a diferença na dotação de fatores (DIF), em termos da razão capital/trabalho, também tem 
impacto positivo sobre o volume de comércio intraindustrial vertical bilateral, corroborando a hipótese central do modelo teórico de Falvey e Kierzkowski (1987).

Tabela 4 - Resultados das estimações econométricas

\section{a) Variável dependente: $\operatorname{Ln} \operatorname{CIIV}_{\mathrm{ijt}}(\alpha=15 \%)$}

\begin{tabular}{ccccc}
\hline & Sinal esperado & MQO (I) & RE (II) & FE (III) \\
\hline Ln PIB & $(+)$ & $3,722^{*}(0,951)$ & $3,231^{*}(0,686)$ & $2,131^{* * *}(1,132)$ \\
Ln DIST & $(-)$ & $-2,162^{* *}(0,926)$ & $-2,133^{* *}(0,952)$ & - \\
Ln DIF & $(+)$ & $0,099(0,325)$ & $0,414^{* * *}(0,255)$ & $0,478(0,312)$ \\
Intercepto $(\alpha 0)$ & & $3,677(8,997)$ & $3,768(10,117)$ & $-8,911(9,700)$ \\
\hline Observações & 290 & 290 & 290 \\
R2 & & 0,51 & 0,50 & 0,43 \\
Teste de Breusch e Pagan & & - & $1.131,43^{*}$ & - \\
Teste de Hausman & & - & 3,82 & - \\
\hline
\end{tabular}

b) Variável dependente: $\operatorname{Ln}$ CIIV $_{\mathrm{ijt}}(\alpha=25 \%)$

\begin{tabular}{ccccc}
\hline & Sinal esperado & MQO (I) & RE(II) & FE(III) \\
\hline Ln PIB & $(+)$ & $3,685^{*}(0,929)$ & $3,211^{* *}(0,668)$ & $2,040^{* * *}(1,120)$ \\
Ln DIST & $(-)$ & $-2,127^{* *}(0,911)$ & $-2,099^{* *}(0,941)$ & - \\
Ln DIF & $(+)$ & $0,100(0,318)$ & $0,426^{* * *}(0,245)$ & $0,498(0,309)$ \\
Intercepto $(\alpha .0)$ & & $3,483(8,869)$ & $3,368(9,806)$ & $-8,606(9,584)$ \\
\hline Observaçőes & & 290 & 290 & 290 \\
R2 & & 0,51 & 0,50 & 0,42 \\
Teste de Breusch e Pagan & & - & $1.114,36^{*}$ & - \\
Teste de Hausman & & \multicolumn{4}{c}{4,04} \\
\hline
\end{tabular}

Fonte: Elaboração própria a partir de estimativas dos autores.

Notas: a) *, **, *** denotam, respectivamente, 1\%, 5\% e 10\% de significância; b) entre parênteses estão reportados os erros-padrão robustos; c) os interceptos $\alpha$ t foram omitidos por economia de espaço.

Os resultados obtidos neste artigo lançam algumas evidências de que existe uma especialização produtiva no comércio intraindustrial, em que o Brasil caracteriza-se como o exportador líquido de produtos de baixa qualidade e importador líquido de produtos de alta qualidade. Em outras palavras, isso significa que a especialização produtiva no comércio internacional pode ocorrer tanto na forma interindustrial e no intercâmbio de produtos homogêneos por produtos diferenciados, quanto na forma intraindustrial, em que, nessa situação, os produtos comercializados são diferenciados conforme as suas qualidades intrínsecas. Ressalta-se que ambas as situações possuem como principal determinante as vantagens comparativas dos países. 
Um ponto importante a salientar é que o tipo de produto comercializado pelo país é de extrema importância para o seu processo de inserção internacional e para o seu desenvolvimento econômico. Fontagné e Freudenberg (2002) apontam que a comercialização dos produtos de alta qualidade possibilita ao país produtor uma melhor inserção no mercado internacional, tendo em vista que esses produtos, de maneira geral, possuem maior elasticidade-renda e menor elasticidade-preço do que os produtos de baixa qualidade. Já Hummels e Klenow (2005) indicam que existe uma relação positiva entre o comércio de produtos de alta qualidade e o crescimento da renda per capita. Sendo assim, os resultados obtidos neste estudo indicam que os policy makers devem desenvolver políticas que visem aprimorar a qualidade do produto brasileiro, melhorando, com isso, a inserção do país no mercado internacional.

\section{Considerações Finais}

Conforme a teoria econômica, o comércio intraindustrial (CII) caracteriza-se quando dois parceiros comerciais trocam simultaneamente produtos de uma mesma origem industrial. Ademais, caso os produtos trocados sejam horizontalmente diferenciados, o comércio intraindustrial será considerado horizontal (CIIH), mas quando os produtos forem verticalmente diferenciados, o comércio intraindustrial será vertical (CIIV). A importância de distinguir esses dois tipos de comércio intraindustrial está relacionada ao fato dos mesmos possuírem diferentes estruturas teóricas. O comércio intraindustrial horizontal (CIIH) é explicado a partir dos modelos de competição monopolística e o comércio intraindustrial vertical (CIIV) é explicado a partir da teoria das vantagens comparativas. Em tese, o primeiro deve ocorrer em maior magnitude entre países que possuem semelhantes níveis de renda, ao passo que o segundo entre países de diferentes níveis de renda.

Inserindo-se nesse contexto, este artigo teve como objetivo principal analisar o comércio intraindustrial (CII) entre o Brasil e os países da OCDE, no período compreendido entre 2000 e 2009. Especificamente, pretendeu-se decompor o comércio intraindustrial (CII) bilateral e analisar os seus determinantes.

Primeiramente, para atingir o objetivo proposto, foi utilizada uma metodologia específica, amplamente difundida na literatura, para mensurar e decompor o comércio intraindustrial (CII) bilateral. Nesse caso, identificou-se que os Estados Unidos e o México são os países da OCDE que possuem o maior volume e índice de comércio intraindustrial com o Brasil, respectivamente. Ademais, verificou-se que, em todas as relações bilaterais, o comércio intraindustrial vertical (CIIV) é superior ao comércio intraindustrial horizontal (CIIH) e que, em geral, os produtos exportados pelo Brasil possuem qualidade inferior à dos produtos importados. 
Após a decomposição do comércio intraindustrial (CII), partiu-se para a análise empírica dos seus determinantes, na qual a hipótese central do modelo econométrico balizou-se no modelo teórico de Falvey e Kierzkowski (1987). De acordo com os resultados obtidos do modelo de efeitos aleatórios, verificou-se que o tamanho do mercado e a distância geográfica têm impactos positivo e negativo sobre o volume de comércio intraindustrial vertical (CIIV) bilateral, respectivamente. Além disso, os resultados também indicaram que a diferença na dotação de fatores, em termos da razão capital/trabalho, tem um efeito positivo sobre o volume de comércio intraindustrial vertical (CIIV) bilateral, corroborando a hipótese do modelo teórico de Falvey e Kierzkowski (1987).

Apesar de todo o esforço metodológico realizado, o presente estudo possui algumas limitações. A principal delas refere-se à utilização do PIB per capita como proxy para a razão capital/trabalho. Nesse caso, apesar dessa ser uma estratégia amplamente utilizada na literatura empírica, considera-se que a utilização de outras proxies, como o estoque de capitais físico e humano, por exemplo, podem trazer novos insights, que corroborem ou que discordem dos resultados obtidos. Outra limitação, a qual é comum nessa literatura, é a falta de um melhor tratamento para os custos do comércio, pois estes tendem a ser mais importantes do que aparentam, conforme argumentam Anderson e Van Wincoop (2004). Adicionalmente, Bergstrand e Egger (2006) enfatizam que diferenças nos custos de comércio entre produtos homogêneos e diferenciados afetam o índice GL e que os efeitos são sensíveis às diferenças nas dotações de recursos.

Uma última limitação que pode servir para uma futura agenda de pesquisa de modo a ampliar os resultados deste artigo diz respeito à própria decomposição do comércio, de modo a evitar o que Azhar e Elliot (2006) chamam de "efeito de proporcionalidade", que se referem às consequências que o escalonamento dos dados pode ter nos efeitos assimétricos nos valores unitários de exportação e importação. Para evitar tal problema, os autores sugerem o uso de um índice GL modificado, o que pode servir como uma possível extensão ao presente artigo.

\section{Referências}

ANDERSON, J.; VAN WINCOOP, E. Trade costs. Journal of Economic Literature, v. 42(3), p. 691-751, 2004.

AZHAR, A. K. M.; ELLIOTT, R. J. On the measurement of produt quality in intra-industry trade. Review of World Economics/Weltwirtschaftliches Archiv, v. 142(3), p. 476-495, 2006.

BALEIX, J. M.; EGIDIO, A. I. M. Intra-industry trade with emergent countries: what can we learn from Spanish data? Economics Bulletin, v. 6, n. 12, p. 1-17, 2005.

BALTAR, C. T. Comércio exterior inter e intra-industrial: Brasil 2003-2005. Economia e Sociedade, Campinas, v. 32, p. 107-134, abr. 2008. 
BERGSTRAND, J. H.; EGGER, P. Trade costs and intra-industry trade. Review of World Economics/Weltwirtschaftliches Archiv, v. 142(3), p. 433-458, 2006.

BLANES, J. V.; MARTIN, C. The nature and causes of intra-industry trade: back to the comparative advantage explanation? The case of Spain. Weltwirtschaftliches Archiv, v. 136, n. 3, p. 423-441, 2000.

CHENG, I.; WALL, H. J. Controlling for heterogeneity in gravity models of trade and integration. Federal Reserve Bank of St. Louis Review, v. 87(1), p. 49-63, Jan./Feb. 2005.

CRESPO, N.; FONTOURA, P. Intra-industry trade by types: what can we learn from Portuguese data? Review of World Economic, v. 140(I), p. 52-79, 2004.

CURZEL, R.; MONTORO, F.; VARTANIAN, P. R. Una investigación de la evolución del comercio intra-industria en la relación Brasil-Mercosur en el periodo 1996-2005: ¿Qué dicen los datos? Revista de Economia Mundial, v. 24, p. 49-66, 2010.

DURKIN, J. T.; KRYGIER, M. Differences in GDP per capita and the share of intra-industry trade: the role of vertically differentiated trade. Review of International Economics, v. 8(4), p. 760-774, 2000.

EKANAYAKE, E. M. et al. I. Intra-industry trade between the United States and Latin America countries. The International Journal of Business and Finance Research, v. 1, n. 2, 2007.

EMIRHAN, P. N. Determinants of vertical intra-industry trade os Turket: panel data approach. Izmir: Dokuz Eylül University, Oct. 2005. (Discussion Paper Series, n. 05/05).

FALVEY, R. E. Commercial policy and intra-industry trade. Journal of International Economics, v. 11, p. 495-511, 1981.

FALVEY, R. E.; KIERZKOWSKI, H. Product quality, intra-industry trade and (im)perfect competition. In: KIERZKOWSKI, H. (Ed.). Protection and Competition in International Trade. Clarendon Press: Oxford, 1987. p. 143-161.

FINGER, J. M. Trade overlap and intra-industry trade. Economic Inquiry, v. 13(4), p. 581-589, 1975.

FLAM, H.; HELPMAN, E. Vertical product differentiation and North-South trade. American Economic Review, v. 77(5), p. 810-822, 1987.

FONTAGNÉ, L.; FREUDENBERG, M. Intra-industry trade: methodological issues reconsidered. Paris: CEPII Research Center, 1997. (CEPII Working Paper, 97-01).

Long-term trends in intra-industry trade. In: LLOYD, P. J.; LEE, H. H. (Ed.). Frontiers of research in intra-industry trade. London: Palgrave Macmillan, 2002.

FONTAGNÉ, L.; FREUDENBERG, M.; GAULIER, G. A systematic decomposition of world trade into horizontal and vertical IIT. Review of World Economics/Weltwirtschaftliches Archiv, v. 142(3), p. 459-475, 2006.

FONTAGNÉ, L.; GAULIER, G.; ZIGNAGO, S. Specialization across varities within products and North-South competition. Paris: CEPII Research Center, 2007. (CEPII Working Paper, 2007-06).

GAULIER, G.; ZIGNAGO, S. BACI: International database at the product-level. Paris: CEPII Research Center, 2010. (CEPII Working Paper, 2010-23). 
GREENAWAY, D.; HINE, R. C; MILNER, C. Country-specific factors and the pattern of horizontal and vertical intra-industry trade in UK. Weltwirtschaftliches Archiv, v. 130(1), p. 77-100, 1994.

GRUBEL, H. G.; LLOYD, P.J. Intra-industry trade. London: Macmillan Press, 1975.

GULLSTRAND, J. Country-specific determinants of vertical intra-industry trade: with application to trade between Poland and EU. In: WAWRZYNJAK, B. (Ed.). Globalisation and change: ways to future. Warsaw: Leon Kozminski Academy of Entrepreneurship and Management, 2000.

HELPMAN, E. Imperfect competition and international trade: evidence from fourteen industrial countries. Journal of the Japanese and International Economics, v. 1(1), p. 62-81, 1987.

HELPMAN, E.; KRUGMAN, P. Market structure and foreign trade. Brighton, UK: Harvester Wheatsheaf, 1985.

HIDALGO, A. B. O intercâmbio comercial brasileiro intra-indústria: uma análise entre indústrias e entre países. Revista Brasileira de Economia, Rio de Janeiro, v. 47, n. 2, p. 243-264, abr./jun. 1993.

HUBER, P. J. The behavior of maximum likelihood estimates under nonstandard conditions. In: BERKELEY SYMPOSIUM ON MATHEMATICAL STATISTICS AND PROBABILITY, 15., 1967, Berkeley. Proceedings... Berkeley: University of California, 1967. v. I, p. 221-33.

HUMMELS, D.; KLENOW, P. The variety and quality of a nation's exports. American Economic Review, v. 95, n. 3, p. 704-723, 2005.

JANSEN, L.; LÜTHJE, T. Driving forces of vertical intra-industry trade in Europe 1996-2005. Review of World Economics, v. 145, p. 469-488, 2009.

KRUGMAN, P. Increasing returns, monopolistic competition and international trade. Journal of International Economics, v. 9(4), p. 469-480, 1979.

Intraindustry specialization and gains from trade. The Journal of Political Economy, v. 89, n. 5 , p. 959-973, Oct. 1981.

Scale economies, product differentiation and the pattern of trade. American Economic Review, v. 70(5), p. 950-959, 1980.

LANCASTER, K. Intra-industry trade under perfect monopolistic competition. Journal of International Economics, v. 10(2), p. 151-170, 1980.

LERDA, S. S. Comércio internacional intra-industrial: aspectos teóricos e algumas evidências, com aplicação ao caso brasileiro. 1988. 171 f. Dissertação (Mestrado em Economia) - Universidade de Brasília, Brasília, DF, 1988.

MACHADO, J. B. M.; MARKWALD, R. A. Dinâmica recente do processo de integração do Mercosul. In: ENCONTRO NACIONAL DE ECONOMIA, 25., 1997, Recife. Anais... Recife: Anpec, 1997. p. 723-742.

MARTIN, J. A.; ORTS, V. Vertical specialization and intra-industry trade: the role of factor endowments. Weltwirtschaftliches Archiv, v. 138(2), p. 340-65, 2002.

NILSSON, L. Two-way trade between unequal partners: the EU and the developing countries. Weltwirtschaftliches Archiv, v. 135(1), p. 102-127, 1999.

RAUCH, E. J. Network versus markets in international trade. Cambridge, MA: National Bureau of Economic Research, June 1996. (NBER Working Paper n. 5617). 
SCHOTT P. K. Across-product versus within-product specialization in international trade. Quarterly Journal of Economics, v. 119(2), p. 647-678, 2004.

STIGLITZ, J. E. The causes and consequences of the dependence of quality of price. Journal of Economic Literature, v. 25, p. 1-48, 1987.

VASCONCELOS, C. R. O comércio Brasil-Mercosul na década de 90: uma análise pela ótica do comércio intra-indústria. Revista Brasileira de Economia, v. 57, p. 283-313, 2003.

WOOLDRIDGE, J. M. Econometric Analysis of Cross Section and Panel Data. Cambridge, MA: MIT Press, 2002.

ZHANG, J.; VAN WITTELOOSTUIJN, A.; ZHOU, C. Chinese bilateral intra-industry trade: a panel data study for 50 countries in the 1992-2001 period. Review of World Economics, v. 141(3), p. 510-540, 2005.

\section{Apêndice A - Estatística Descritiva das Variáveis}

\begin{tabular}{|c|c|c|c|c|c|c|}
\hline Variável & & $\begin{array}{l}\text { Número de } \\
\text { observações }\end{array}$ & Média & $\begin{array}{l}\text { Desvio- } \\
\text { padrão }\end{array}$ & Mínimo & Máximo \\
\hline \multirow[t]{3}{*}{$\operatorname{Ln} \operatorname{CIIV}(\alpha=15 \%)$} & Overal & 290 & 10,779 & 1,029 & 1,029 & 16,015 \\
\hline & Between & 29 & & 2,384 & 2,384 & 15,451 \\
\hline & Within & 10 & & 8,190 & 8,190 & 12,677 \\
\hline \multirow[t]{3}{*}{$\operatorname{Ln} \operatorname{CIIV}(\alpha=25 \%)$} & Overal & 290 & 10,675 & 2,478 & 1,029 & 15,896 \\
\hline & Between & 29 & & 2,435 & 2,384 & 15,342 \\
\hline & Within & 10 & & 0,630 & 7,894 & 12,643 \\
\hline \multirow[t]{3}{*}{ Ln PIB } & Overal & 290 & 7,762 & 0,479 & 7,123 & 9,702 \\
\hline & Between & 29 & & 0,457 & 7,360 & 9,525 \\
\hline & Within & 10 & & 0,162 & 7,498 & 8,051 \\
\hline \multirow[t]{3}{*}{ Ln DIST } & Overal & 290 & 9,192 & 0,207 & 8,908 & 9,797 \\
\hline & Between & 29 & & 0,210 & 8,908 & 9,797 \\
\hline & Within & 10 & & 0,000 & 9,192 & 9,192 \\
\hline \multirow[t]{3}{*}{ Ln DIF } & Overal & 290 & 9,739 & 0,793 & 5,975 & 11,596 \\
\hline & Between & 29 & & 0,778 & 7,385 & 11,421 \\
\hline & Within & 10 & & 0,204 & 8,329 & 10,922 \\
\hline
\end{tabular}

Fonte: Elaboração própria a partir da base de dados da pesquisa.

Recebido em: 13/07/2012.

Aceito em: 31/01/2013. 